\title{
The Effect of Preceding Sequences on Stock Returns
}

\author{
Andrey Kudryavtsev ${ }^{*}$
}

\begin{abstract}
This study explores the effect of the gambler's fallacy on stock returns. I hypothesize that if during a number of consecutive trading days, a stock's return is positive (negative), then due to the gambler's fallacy, at least some of the investors may believe that the stock's price "has" to subsequently fall (rise), and thus, to increase their willingness to sell (buy) the stock, resulting in negative (positive) abnormal market-adjusted stock returns. Employing a large sample of daily stock price data, I was able to document that following relatively long sequences of positive (negative) stock returns, abnormal stock returns are on average significantly negative (positive), indicating the existence of the price pressure towards the return sign reversal. Moreover, the magnitude of the effect is stronger for longer return sequences. The effect is found to be more pronounced for smaller and more volatile stocks, and is robust to other relevant company - and stockspecific factors.
\end{abstract}

Key words: Abnormal Stock Returns; Gambler's Fallacy; Investment Decisions; Price Reversals; Stock Return Sequences.

JEL classification: G11, G12, G19.

\section{Introduction}

Stock prices and returns have traditionally attracted enormous attention of both stock market researchers and practitioners. Numerous attempts to explain and predict stock performance have been made, by employing a wide range of techniques, methods and explanatory factors. In the recent decades, an increasing number of stock market studies explicitly account for the fact that investors are human beings, and detect various psychological factors that appear to exert significant influence on investors' ways of making decisions, and consequently, on the stock prices. The present study contributes to this strand of literature and sheds light on the effect of the gambler's fallacy on stock returns.

The gambler's fallacy was first documented by Laplace (1796). This psychological bias refers to a belief in negative autocorrelation of random non-autocorrelated sequences. A classic example comes from the world of casino, where a person who is affected by the gambler's fallacy may expect that following a sequence of four black numbers appearing on the roulette wheel, a red number "has" to appear,

Andrey Kudryavtsev; Economics and Management Department, The Max Stern Yezreel Valley Academic College, Emek Yezreel 19300, Israel, <andreyk@yvc.ac.il>. 
that is, its probability becomes higher than that of a black one. In this respect, I expect that if during a number of consecutive trading days, a stock's return is positive (negative), then because of the gambler's fallacy, at least some of the investors may believe that the stock's price "has" to subsequently fall (rise). Subsequently, these investors' willingness to sell (buy) the stock may increase, creating a selling (buying) pressure, which is beyond the one rationally motivated by the stock's fundamentals and future profit potential. In other words, I expect that excess market-adjusted stock returns should be, on average, lower (higher) following relatively long sequences of positive (negative) stock returns. In addition, I suggest that, similarly to a casino where people tend to place more bets on a red number following longer sequences of black numbers appearing on the wheel, in the stock market, the longer the sequence of trading days with the samesign return for the respective stock, the stronger may be the investors' inclination to expect the reversal in the sign of the stock's return, leading to the price pressure in the direction of the reversal.

Employing the daily stock price data for all the stocks that made up S\&P 500 Index during the years 1990 to 2016, I documented a corroborative evidence for both above-mentioned research hypotheses. Firstly, I find that after the sequences of different length of positive (negative) stock returns, abnormal stock returns are on average significantly negative (positive), indicating the existence of the price pressure towards the return sign reversal, which on average indeed leads to the reversal. This effect appears to be slightly more pronounced following the sequences of positive, compared to the respective sequences of negative, stock returns. This possibly indicates that the fear of the stock price decrease following a sequence of positive-return days is stronger than the hope for the stock price increase following a sequence of negative-return days. Secondly, the absolute values of average and median ARs, as well as the percentage of days ended up with return reversals, gradually and significantly increase with the length of the preceding sequence, suggesting that the tendency for return sign reversal is enhanced by longer sequences of the same-sign returns. Furthermore, in line with the previous literature dealing with psychological effects on investors' behaviour, I detect that the effect of preceding sequences on stock returns is significantly more pronounced for smaller and more volatile stocks, suggesting that in the cases when investors possess a relatively smaller amount of relevant information about the stocks they trade, they are more inclined to apply simplifying decision-making techniques. Finally, by running a multifactor regression, I documented that the effect of preceding sequences on stock returns persists and remains significant after controlling for other potentially influential factors, including contemporaneous market returns, firms' market capitalization, Market-Model beta, and historical stock returns and volatilities. 
The rest of the paper is structured as follows. Section 2 reviews the literature dealing with the gambler's fallacy and its economic and financial implications. In Section 3, I formulate and explain the study's research hypotheses. Section 4 describes the database employed in this study. Section 5 introduces the empirical tests and presents the results. Section 6 concludes and provides a brief discussion.

\section{Literature Review: Gambler's Fallacy, its Causes and Implications}

Previous literature formally defines the gambler's fallacy as a belief in negative autocorrelation of random non-autocorrelated sequences. For example, individuals affected by the gambler's fallacy may expect that after four black numbers appearing on the roulette wheel, a red number "has" to appear, or in other words, its probability becomes higher than that of a black number.

Gambler's fallacy is first described by Laplace (1796). The respective biased beliefs are first detected in the laboratory experiments dealing with probability matching. The subjects taking part in these experiments are asked to guess the colour of the light would illuminate next, and after observing a string of one of the colours, they tend to guess the other colour is going to illuminate. The literature refers to this effect as negative recency (see Estes, 1964, and Lee, 1971, for reviews). Ayton and Fischer (2004) reveal similar beliefs when asking subjects to choose which of two colours will appear next on a roulette wheel. Gal and Baron (1996) demonstrate that the behaviour which is consistent with the gambler's fallacy has deeper roots than a simply boredom. The authors ask their experiment participants to try and maximize their earnings, and get responses that are in line with the gambler's fallacy approach.

The commonly accepted psychological cause for the gambler's fallacy is the representativeness heuristic (Tversky and Kahneman, 1971; Kahneman and Tversky, 1972). People are suggested to perceive random sequences as "a selfcorrecting process in which a deviation in one direction induces a deviation in the opposite direction to restore the equilibrium" (Tversky and Kahneman, 1974, p. 1125). Therefore, following a sequence of three black numbers appearing on the wheel, a red number seems more likely to occur, since a sequence "black-blackblack-red" looks more representative of the underlying distribution than a sequence "black-black-black-black".

A number of previous studies empirically reveal the gambler's fallacy in gambling. For example, Clotfelter and Cook (1991, 1993) and Terrell (1994) detect that during a period of time after a given lottery number wins, people tend to place less bets on it. Following a period of several months, the winning number becomes a 'usual' one with regular betting patterns. Metzger (1984), Terrell and Farmer (1996) and Terrell (1998) analyse the data from horse and dog racing, and 
document similar patterns. Croson and Sundali (2005) and Sundali and Croson (2006) employ real betting data from a casino and document a behaviour which is consistent with the gambler's fallacy. Namely, after a sequence of outcomes of one of the colours, people tend to bet more on the other colour.

Zielonka (2004) performs a survey in order to uncover how stock market professionals make their trading decisions, and finds that the rules and approaches of the technical analysis have much in common with several behavioural biases, including the gambler's fallacy. Goetzmann and Kumar (2008) argue that U.S. investors who exhibit trend-contrarian behaviour tend to perform less portfolio diversification. This kind of behaviour has negative consequences from the point of view of both risk and performance parameters. Kudryavtsev et al. (2013) experimentally quantify the extent to which each specific stock market investor is affected by a number of behavioural biases, including the gambler's fallacy. They suggest that the degrees of the biases are positively correlated in the cross-section, or in other words, that investors who accept a given (biased) decision-making rule, tend to agree with other ones, as well.

\section{Research Hypotheses}

As discussed in the previous Section, the gambler's fallacy may cause people to expect that after a sequence of instances in which a certain outcome has been realized, the probability of the opposite outcome's realization increases. Stock market investors, as human beings, might also share this belief. Assuming that this may be true, one may expect that if during a number of consecutive trading days, a stock's return is positive (negative), then at least some of the investors may expect that the stock's price "has" to fall (rise), so that their willingness to sell (buy) the stock increases, creating a selling (buying) pressure, which is beyond the one rationally motivated by the stock's fundamentals and future profit potential. Furthermore, similarly to a casino where the longer sequences of red numbers appearing on the wheel increase even more the willingness to bet on a black number, the longer the sequence of trading days with the same-sign return for the respective stock, the stronger may be the investors' inclination to expect the reversal in the sign of the stock's return, leading to the price pressure in the direction of the reversal.

Therefore, I hypothesize that, all other things being equal:

Hypothesis 1: A stock's excess market-adjusted return should be lower (higher) following a number of consecutive trading days when the stock's return was positive (negative). 
Hypothesis 2: The decrease (increase) in the stock's excess market-adjusted return should be more pronounced the longer the preceding sequence of the positive (negative)-return days.

In other words, I expect that relatively long sequences of daily stock returns of the same-sign may create expectations for the return reversals that finally end up with the reversals, or at least, push the stock returns towards the reversals. It should be emphasized that on the one hand, when potentially creating their casino-like beliefs in further stock price reversals investors may consider sequences of raw daily stock returns, but on the other hand, when testing for the effect of these beliefs one has to analyse the excess market-adjusted stock returns. I furthermore test for the existence of this "preceding sequence" effect on stock returns.

\section{Data Description}

In my empirical analysis, I employ the adjusted daily stock price data for all the constituents of S\&P 500 Index as of December 31, 2016, as recorded at www.finance.yahoo.com. The sampling period for each given stock starts on January 1, 1990 or at the first day of the stock's trading history reported by the website, and ends on December 31, 2016, yielding an overall sample of 2,425,650 stock-days. Daily values of the S\&P 500 Index, which I use as a proxy for the general stock market index, are downloaded from the same website.

For each trading day $t$, I calculate abnormal, or excess, stock returns (ARs) for each stock $i$, according to the Market Model, that is:

$$
A R_{i t}=S R_{i t}-\text { Alpha }_{i t}-\text { Beta }_{i t} M R_{t},
$$

where: $A R_{i t}$ is stock $i$ 's abnormal return on day $t ; S r_{i t}$ is stock i's log return on day $t ; M R_{t}$ is the market index (S\&P 500) log return on day $t$; and Alpha $a_{i t}$, Beta $_{i t}$ are the Market-Model parameters for stock $i$ corresponding to day $t$, estimated by regressing the stock's returns on the contemporaneous market returns over 250 trading days (approximately one year) preceding day $t$. In other words, separately for each trading day $t$, I estimate the parameters Alpha $a_{i t}$, Beta $_{i t}$ for the stock $i$ based on the univariate OLS regression of the stock's returns on market returns over the preceding estimation period. This moving regression approach leads to the fact that the stock parameters for each given stock change over time ${ }^{1}$.

Finally, for each day $t$, I match the underlying firm's market capitalization, as recorded on a quarterly basis at http://ycharts.com/, for the closest preceding date.

1 Alternatively, I calculate ARs using Market Adjusted Returns (MAR) - return differences from the market index, and the Fama-French three-factor plus momentum model. The results (available upon request from the author) remain qualitatively similar to those reported in Section 5. 


\section{Research Methodology and Results}

\subsection{The effect of preceding sequences on stock returns: Comparative analysis}

Firstly, I calculate abnormal stock returns following sequences of days when the respective stock's returns had the same sign. Having in mind both research hypotheses, I define a number of alternative return sequence lengths, namely: (i) three days; (ii) four days; (iii) five days; (iv) six days; (v) seven days or more. Since the effect of preceding return sequences on stock returns probably appears during the trading day when the stock return changes its sign, I append the zeroreturn days to the sequences. For example, if a stock's returns were negative during two consecutive days, zero on the third day and negative again on the forth day, I assume that the sequence of negative returns was not interrupted, and consider the stock's abnormal returns following the sequences of three, four and five positive-return trading days. Once again, it should be emphasized that when defining the sequences of days with the same-sign returns, I consider the raw daily stock returns, which are those that may potentially create a ground for the investors' beliefs in further price reversals.

Table 1 presents basic descriptive AR statistics for the days following positive and negative return sequences of different length, and their statistical significance. The results corroborate both research hypotheses. First, consistently with Hypothesis 1, following the sequences of all lengths of positive (negative) stock returns, stock ARs are on average significantly negative (positive), indicating the existence of the price pressure towards the return sign reversal, which on average indeed leads to the reversal ${ }^{2}$. Secondly, in line with Hypothesis 2, the absolute values of average and median ARs, as well as the percentage of days ended up with return reversals, gradually and significantly increase with the length of the preceding sequence, suggesting that the tendency for return sign reversal is enhanced by longer sequences of the same-sign returns. For example, average ARs following the three-day sequences of positive (negative) stock returns equal $-0.274 \%$ $(0.234 \%)$, compared to $-0.543 \%(0.485 \%)$ following the sequences of seven or more days. An additional observation is that the effect of the preceding sequences on stock returns is slightly more pronounced following the sequences of positive returns. It possibly suggests that the fear of the stock price decrease following a sequence of positive-return days is stronger than the hope for the stock price increase following a sequence of negative-return days.

\footnotetext{
As a robustness check, I have compared the average AR returns following the sequences of the same-sign stock returns to the average values of the series of both raw and abnormal stock returns randomly drawn from the study's total sample. The results (available upon request from the author) indicate that average ARs reported in Table 1 significantly differ from the average values of the random series.
} 


\section{Tab. 1: Descriptive statistics of abnormal stock returns (ARs) following sequences of the same-sign stock returns}

\begin{tabular}{|c|c|c|c|c|c|}
\hline \multicolumn{6}{|c|}{ Panel A: AR statistics following the sequences of positive stock returns } \\
\hline \multirow[b]{2}{*}{ Statistic measures } & \multicolumn{5}{|c|}{ Preceding sequence length } \\
\hline & $\begin{array}{l}3 \text { days } \\
(146,375 \\
\text { sequences })\end{array}$ & $\begin{array}{l}4 \text { days } \\
(61,990 \\
\text { sequences })\end{array}$ & $\begin{array}{l}5 \text { days } \\
(25,813 \\
\text { sequences })\end{array}$ & $\begin{array}{l}6 \text { days } \\
(10,338 \\
\text { sequences })\end{array}$ & $\begin{array}{l}7 \text { days or } \\
\text { more } \\
(4,007 \\
\text { sequences) }\end{array}$ \\
\hline Average, $\%$ & $*_{-}-0.274$ & $* *-0.342$ & $* * *-0.389$ & $* * *-0.461$ & $* * *-0.543$ \\
\hline Median, \% & $*_{-0.264}$ & $*_{-0} 0.331$ & $*_{-0} 0.374$ & $* * *_{-} 0.447$ & $* * *_{-}-0.521$ \\
\hline Standard deviation, $\%$ & 1.105 & 1.114 & 1.118 & 1.132 & 1.157 \\
\hline Percent of positive & 42.35 & 41.64 & 40.05 & 38.76 & 36.29 \\
\hline
\end{tabular}

Panel B: AR statistics following the sequences of negative stock returns

Preceding sequence length

\begin{tabular}{llllll} 
Statistic measures & $\mathbf{3}$ days & $\mathbf{4}$ days & $\mathbf{5}$ days & $\mathbf{6}$ days & $\begin{array}{l}\mathbf{7} \text { days or } \\
\text { more }\end{array}$ \\
& $\begin{array}{l}(\mathbf{1 4 2 , 8 3 9} \\
\text { sequences })\end{array}$ & $\begin{array}{l}\mathbf{( 6 2 , 1 7 8} \\
\text { sequences) }\end{array}$ & $\begin{array}{l}\mathbf{( 2 6 , 4 6 9} \\
\text { sequences) }\end{array}$ & $\begin{array}{l}\mathbf{( 1 1 , 0 9 3} \\
\text { sequences) }\end{array}$ & $\begin{array}{l}\mathbf{( 4 , 4 5 2} \\
\text { sequences) }\end{array}$ \\
\hline Average, \% & $* 0.234$ & $* 0.285$ & $* * 0.343$ & $* * * 0.409$ & $* * * 0.485$ \\
Median, \% & $* 0.219$ & $* 0.268$ & $* * 0.331$ & $* * 0.387$ & $* * * 0.459$ \\
Standard deviation, \% & 1.075 & 1.086 & 1.093 & 1.108 & 1.120 \\
Percent of positive & 56.47 & 57.43 & 58.09 & 59.87 & 61.08 \\
\hline
\end{tabular}

Source: www.finance.yahoo.com + authorial computation.

Note: Asterisks denote 2-tailed p-values: ${ }^{*} \mathrm{p}<0.10 ; * * \mathrm{p}<0.05 ; * * * \mathrm{p}<0.01$

(H0: Average/Median AR =0).

Following the above findings, I empirically test for the long-term profitability of a strategy based on the expectations of stock price reversals after the sequences of days with the same-sign returns. Since the strongest evidence of reversals is detected following the sequences of seven or more days, I consider the strategy which builds for each trading day an equally-weighted long (short) portfolio of stocks with seven or more days of negative (positive) returns, and adjusts this portfolio on a daily basis. Assuming that an investor employing this strategy pays buying and selling fee of $0.05 \%$ of the traded value, I find that over the period from 1990 through 2016, she earns an average yearly return of $16.68 \%$, which is significantly higher than the average yearly return of the S\&P 500 Index over the same period, accounting to $7.01 \%$. 


\subsection{The effect of preceding sequences on stock returns: Subsample analysis}

Having documented the effect of preceding sequences on stock returns for the total sample, I now verify if the magnitude of the effect may differ for different groups of stocks. The motivation for this analysis arises from the previous literature dealing with the effects of various behavioural biases on investors' decisions. A number of studies in this field (e.g., Baker and Wurgler, 2006; Kliger and Kudryavtsev, 2010) conclude that - small stocks, high volatility stocks, extreme growth stocks, younger stocks, non-dividend paying stocks, unprofitable stocks, , and distressed stocks - are more likely to be affected by psychological biases. In other words, stocks preferred by optimists and speculators and neglected by arbitrageurs are more sensitive to various irrational psychological effects. Following these findings, I first divide my working sample in subsamples according to the firm size. For each trading day during the sampling period, I split the total sample into three roughly equal parts by the firms' market capitalization (low, medium and high) reported for the end of the preceding quarter, and then calculate average stock ARs following the sequences of the same-sign returns separately for the different size groups. Table 2 contains the respective average AR measures and their statistical significance. The results are clearly consistent with the previous literature, indicating that the effect of preceding sequences on stock returns is significantly more pronounced for smaller stocks. For example, average ARs following the sequences of seven or more days of positive (negative) stock returns equal $-0.466 \%(0.420 \%)$ for high capitalization stocks, compared to $0.632 \%(0.557 \%)$ for low capitalization stocks.

\section{Tab. 2: Average abnormal stock returns (ARs) following sequences of the same-sign stock returns: The sequences are classified by the firms' market capitalization}

\begin{tabular}{|c|c|c|c|c|c|}
\hline \multicolumn{6}{|c|}{ Panel A: Average ARs, \% for the days following the sequences of positive stock returns } \\
\hline \multirow{2}{*}{ Stock categories } & \multicolumn{5}{|c|}{ Preceding sequence length } \\
\hline & 3 days & 4 days & 5 days & 6 days & 7 days or more \\
\hline Low capitalization & $* *-0.385$ & $* * *_{-} 0.412$ & $* * *-0.476$ & $* * *-0.534$ & $* * *_{-} 0.632$ \\
\hline $\begin{array}{l}\text { Medium } \\
\text { capitalization }\end{array}$ & $*_{-} 0.258$ & $*_{*}-0.334$ & $* *_{-} 0.375$ & $* * *_{-} 0.448$ & $* * *-0.531$ \\
\hline High capitalization & -0.179 & $*_{-} 0.280$ & $*-0.316$ & $*_{-}^{*}-0.401$ & $* *-0.466$ \\
\hline
\end{tabular}


Panel B: Average ARs, \% for the days following the sequences of negative stock returns

Preceding sequence length

\begin{tabular}{llllll} 
Stock categories & $\mathbf{3}$ days & $\mathbf{4}$ days & $\mathbf{5}$ days & $\mathbf{6}$ days & $\begin{array}{l}\mathbf{7} \text { days or } \\
\text { more }\end{array}$ \\
\hline Low capitalization & $* * 0.289$ & $* * 0.359$ & $* * * 0.397$ & $* * * 0.468$ & $* * * 0.557$ \\
Medium capitalization & $* 0.221$ & $* 0.276$ & $* * 0.344$ & $* * 0.407$ & $* * * 0.478$ \\
High capitalization & 0.192 & $* 0.220$ & $* 0.288$ & $* * 0.352$ & $* * 0.420$ \\
\hline
\end{tabular}

Source: www.finance.yahoo.com + authorial computation.

Note: Asterisks denote 2-tailed p-values: $* \mathrm{p}<0.10 ; * * \mathrm{p}<0.05 ; * * * \mathrm{p}<0.01$

(H0: Average AR =0).

Furthermore, I classify my sample according to the stocks' historical volatility. For each trading day during the sampling period, I split the total sample into three roughly equal parts by the standard deviation of stock returns over 250 preceding trading days (low, medium and high volatility stocks). Table 3 reports average ARs and their significance separately for the three subsamples. Once again, in line with the previous literature, the effect of preceding sequences on stock returns appears to be significantly more pronounced for more volatile stocks. For example, average ARs following the sequences of seven or more days of positive (negative) stock returns equal $-0.599 \%(0.543 \%)$ for high volatility stocks, compared to $-0.487 \%(0.426 \%)$ for low volatility stocks.

Overall, the results in this subsection look well expected and quite intuitive. They suggest that in the cases when investors possess a relatively smaller amount of relevant information about the stocks they trade (small and volatile stocks), they are more inclined to apply simplifying decision-making techniques, which, among other psychological biases, may lead to the effect of preceding sequences on stock returns.

\section{Tab. 3: Average abnormal stock returns (ARs) following sequences of the same-sign stock returns: The sequences are classified by the stocks' historical volatility}

Panel A: Average ARs, \% for the days following the sequences of positive stock returns

\begin{tabular}{|c|c|c|c|c|c|}
\hline \multirow{2}{*}{$\begin{array}{l}\text { Stock } \\
\text { categories }\end{array}$} & \multicolumn{5}{|c|}{ Preceding sequence length } \\
\hline & 3 days & 4 days & 5 days & 6 days & 7 days or more \\
\hline Low volatility & -0.196 & $*_{-0.293}$ & $*_{-} 0.325$ & $* *_{-0} 0.408$ & $* * *_{-} 0.487$ \\
\hline $\begin{array}{l}\text { Medium } \\
\text { volatility }\end{array}$ & $*_{-0} 0.264$ & $*_{*}-0.342$ & $*_{*}-0.379$ & $* * *_{-}-0.457$ & $* * *-0.543$ \\
\hline High volatility & $* *-0.362$ & $*_{*}-0.391$ & $* * *_{-} 0.463$ & $* * *_{-} 0.518$ & $* * *_{-} 0.599$ \\
\hline
\end{tabular}


Kudryavtsev, A.: The Effect of Preceding Sequences on Stock Returns.

Panel B: Average ARs, \% for the days following the sequences of negative stock returns

\begin{tabular}{llllll}
\hline \multirow{2}{*}{ Stock categories } & \multicolumn{6}{l}{ Preceding sequence length } & & \\
& $\mathbf{3}$ days & $\mathbf{4}$ days & $\mathbf{5}$ days & $\mathbf{6}$ days & $\mathbf{7}$ days or more \\
\hline Low volatility & 0.194 & $* 0.228$ & $* 0.295$ & $* * 0.362$ & $* * 0.426$ \\
Medium volatility & $* 0.233$ & $* 0.281$ & $* * 0.353$ & $* * 0.414$ & $* * * 0.486$ \\
High volatility & $* * 0.275$ & $* * 0.346$ & $* * * 0.381$ & $* * * 0.451$ & $* * * 0.543$ \\
\hline
\end{tabular}

Source: www.finance.yahoo.com + authorial computation.

Note: Asterisks denote 2-tailed p-values: *p<0.10; **p<0.05; ***p<0.01

(H0: Average AR =0).

\subsection{The effect of preceding sequences on stock returns: Multifactor regression analysis}

At this stage, I proceed to testing if the effect of preceding sequences on stock returns remains significant if other potentially influential factors are controlled for. In order to do that, I run the following regression based on the panel data of stock returns over the sampling period:

$$
\begin{aligned}
& A R_{i t}=\alpha_{i}+\beta_{1 i} P O S 3_{i t}+\beta_{2 i} P O S 4_{i t}+\beta_{3 i} P O S 5_{i t}+\beta_{4 i} P O S 6_{i t}+ \\
& +\beta_{5 i} \text { POS7 plus } \text { it }+\beta_{6 i} N E G 3_{i t}+\beta_{7 i} N E G 4_{i t}+\beta_{8 i} N E G 5_{i t}+\beta_{9 i} N E G 6_{i t}+\text {, } \\
& +\beta_{10 i} N E G 7 \text { plus }_{i t}+\beta_{11 i} S_{i t-1}+\beta_{12 i} M_{i t}+\beta_{13 i} \text { MCap }_{i t}+\beta_{14 i} \text { Beta }_{i t}+ \\
& +\beta_{15 i} \mathrm{CumSR}_{i t}+\beta_{16 i} \mathrm{STDevSR} R_{i t}+\varepsilon_{i t}
\end{aligned}
$$

where: $P O S 3_{i t}$ to POS7 plus $_{i t}$ are the dummy variables, taking the value 1 if for stock $i$, day $t$ was preceded by three to seven or more days of positive returns, respectively, and 0 otherwise; $N E G 3^{\text {it }}$ to $N E G 7$ plus $_{i t}$ are the dummy variables, taking the value 1 if for stock $i$, day $t$ was preceded by three to seven or more days of negative returns, respectively, and 0 otherwise; $M C a p_{i t}$ is the natural logarithm of firm $i$ 's market capitalization for the end of the quarter preceding day $t ;$ Beta $_{i t}$ is stock $i$ 's Market-Model beta estimated over 250 trading days preceding day $t$; $\mathrm{CumSR}_{i t}$ is stock $i$ 's cumulative return over 250 trading days preceding day $t$; and $S T D e v S R_{i t}$ is the standard deviation of stock $i$ 's returns over 250 trading days preceding day $t$.

Table 4 reports some basic descriptive statistics of the explanatory companyspecific variables, while Table 5 comprises the results of regression (2), including the coefficient estimates and their statistical significance. The results indicate that:

- The coefficient estimates of all the dummy variables related to the preceding sequences of positive (negative) returns are negative (positive) and highly statistically significant. This represents a strong support for Hypothesis 1, demonstrating that preceding sequences of positive (negative)-return days tend 
to decrease (increase) the subsequent stock returns, and this effect is not driven by other relevant company-specific factors.

- In line with Hypothesis 2, for both positive and negative return sequences, the absolute values of the coefficient estimates of the sequence dummies significantly increase following longer return sequences.

- Consistently with subsection 5.1, the absolute values of the coefficient estimates of POS dummies are slightly higher than those of the respective NEG ones, indicating that the preceding sequence effect on stock returns is slightly more pronounced following positive return sequences.

Tab. 4: Descriptive statistics of company-specific variables

\begin{tabular}{llll}
\hline Variables & Mean & Median & $\begin{array}{l}\text { Standard } \\
\text { Deviation }\end{array}$ \\
\hline SR, \% & 0.021 & 0.020 & 0.876 \\
MCap & 22.39 & 20.37 & 7.32 \\
Beta & 1.08 & 1.07 & 0.45 \\
CumSR, \% & 5.127 & 5.023 & 12.671 \\
\hline
\end{tabular}

Source: www.finance.yahoo.com + authorial computation.

Tab. 5: Regression analysis: Preceding sequence effect on stock returns (Dependent variable - AR, \%)

\begin{tabular}{ll}
\hline Explanatory variables & Coefficient estimates (t-statistics) \\
\hline Intercept & $* * * 0.012(2.98)$ \\
POS3t & $* *-0.196(-2.12)$ \\
POS4t & $* * *-0.245(-2.56)$ \\
POS5t & $* * *-0.296(-3.21)$ \\
POS6t & $* * *-0.357(-4.09)$ \\
POS7plust & $* * *-0.432(-4.89)$ \\
NEG3t & $* * 0.175(1.98)$ \\
NEG4t & $* * 0.221(2.30)$ \\
NEG5t & $* * * 0.277(2.84)$ \\
NEG6t & $* * * 0.320(3.42)$ \\
NEG7plust & $* * * 0.384(4.13)$ \\
SRt-1 & $-0.037(-0.76)$ \\
MRt & $* 0.117(1.74)$ \\
\hline
\end{tabular}




\begin{tabular}{ll}
\hline Explanatory variables & Coefficient estimates (t-statistics) \\
\hline MCapt & $* 0.034(1.85)$ \\
Betat & $0.231(0.97)$ \\
CumSRt & $0.061(1.25)$ \\
STDevSRt & $0.033(0.47)$ \\
\hline Adjusted R-squared & $\mathbf{0 . 2 3 5}$ \\
\hline
\end{tabular}

Source: www.finance.yahoo.com + authorial computation.

Note: Asterisks denote 2-tailed p-values: ${ }^{*} \mathrm{p}<0.10 ; * * \mathrm{p}<0.05 ; * * * \mathrm{p}<0.01$.

\section{Conclusion and Discussion}

In this study, I make an effort to contribute to the rapidly developing strand of literature which deals with behavioural factors affecting stock prices. Namely, I hypothesize that investors' decisions to buy or sell stocks may be affected by the gambler's fallacy, and if so, following a number of consecutive trading days characterized by positive (negative) returns for a given stock, its abnormal marketadjusted returns should be, on average, negative (positive), and even more so, the longer the preceding return sequence.

I employ a large sample of daily stock price data, and find support for the study's research hypotheses. I establish that after the sequences of different length of positive (negative) stock returns, abnormal stock returns tend to be negative (positive), indicating the existence of the price pressure towards the return sign reversal. Secondly, the magnitude of the effect of preceding sequences on stock returns gradually and significantly increases with the return sequence length, and is higher for low capitalization and highly volatile stocks. The effect remains significant after controlling for additional company-specific and market-wide factors. Importantly, I establish that the trading strategy based on the expectation of stock price reversals after the sequences of days characterized by the same-sign returns is expected to yield average returns which are significantly higher than those yielded by the buy-and-hold strategy employing the S\&P 500 Index. This finding contradicts the efficient market hypothesis, suggesting that psychological effects may potentially create non-negligible investment opportunities.

This research has yielded a series of results of potential practical relevance. The results imply that investors' trading activity, which is suggested to be a multi-level and complicated mechanism, may be also influenced by psychological biases, including the gambler's fallacy. Further research might invest efforts in analysing the effect for different sectors and categories of stocks, different countries, shorter (intraday) and longer (weekly, monthly) time intervals, and different 
macroeconomic backgrounds, including the periods of financial crises. The further research could be also aimed at putting this study's findings in a broader perspective, since the former may be potentially connected to a series of other topics:

- There exist several technical strategies implicitly looking for both intraday and inter-day stock price reversals, and it might be worth analysing in which cases their recommendations may be consistent or contradict the findings of this study, and what the investment performance associated with the respective recommendations is expected to be.

- Though the local stock price fluctuations are not supposed to be those that lead to serious deviations of the stock prices from their fundamental values, it still might be interesting to introduce some fundamental stock valuation criteria in the analysis presented in this study, in order to establish if the tendency for the stock price reversals of the above-described nature is amplified when the stock prices deviate from the fundamentals and "strive" to get back to them.

- If the stock holdings and trading data for individual investors are available, one could analyse if trading strategies based on the expectations of reversals of the above-described nature increase or decrease the respective investors' portfolio risk, and also if these expectations are self-fulfilling because of the effect of herding.

\section{References}

Ayton, P., Fischer, I., 2004. The hot hand fallacy and the gambler's fallacy: Two faces of subjective randomness? Memory and Cognition 32, 1369-1378. DOI: 10.3758/BF03206327.

Baker, M., Wurgler, J., 2006. Investor sentiment and the cross-section of stock returns. Journal of Finance 61, 1645-1680. DOI: 10.1111/j.15406261.2006.00885.x.

Clotfelter, C., Cook, P., 1991. Lotteries in the real world. Journal of Risk and Uncertainty 4, 227-232. DOI: 10.1007/BF00114154.

Clotfelter, C., Cook, P., 1993. The gambler's fallacy in lottery play. Management Science 39, 1521-1525. DOI: 10.1287/mnsc.39.12.1521.

Croson, R., Sundali, J., 2005. The gambler's fallacy and the hot hand: Empirical data from casinos. Journal of Risk and Uncertainty 30, 195-209. DOI: 10.1007/s11166-005-1153-2.

Estes, W., 1964. Probability learning. In A.W. Melton (ed.), Categories of Human Learning, Academic Press. New York. 
Gal, I., Baron, J., 1996. Understanding repeated simple choices. Thinking and Reasoning 1, 81-98. DOI: 10.1080/135467896394573.

Goetzmann, W. N., Kumar, A., 2008. Equity portfolio diversification. Review of Finance 12, 433-463. DOI: 10.1093/rof/rfn005.

Kahneman, D., Tversky, A., 1972. Subjective probability: A judgment of representativeness. Cognitive Psychology 3, 430-454. DOI: 10.1016/00100285(72)90016-3.

Kliger, D., Kudryavtsev, A., 2010. The availability heuristic and investors' reaction to company-specific events. Journal of Behavioral Finance 11, 50-65. DOI: $10.1080 / 15427561003591116$.

Kudryavtsev, A., Cohen, G., Hon-Snir, S., 2013. "Rational" or "intuitive": Are behavioral biases correlated across stock market investors? Contemporary Economics 7, 31-53. DOI: 10.5709/ce.1897-9254.81.

Laplace, P. S., 1951. A philosophical essay on probabilities. Dover, New York. (Original work published in 1796).

Lee, W., 1971. Decision theory and human behavior. Wiley, New York.

Metzger, M., 1984. Biases in betting: An application of laboratory findings. Psychological Reports 56, 883-888. DOI: 10.2466/pr0.1985.56.3.883.

Sundali, J., Croson, R., 2006. Biases in casino betting: The hot hand and the gambler's fallacy. Judgment and Decision Making 1, 1-12.

Terrell, D., 1994. A test of the gambler's fallacy: Evidence from pari-mutuel games. Journal of Risk and Uncertainty 8, 309-317. DOI: 10.1007/BF01064047.

Terrell, D., 1998. Biases in Assessments of Probabilities: New Evidence from Greyhound Races. Journal of Risk and Uncertainty 17, 151-166.

Terrell, D., Farmer, A., 1996. Optimal betting and efficiency in pari-mutuel betting markets with information costs. The Economic Journal 106, 846-868.

Tversky, A., Kahneman, D., 1971. Belief in the law of small numbers. Psychological Bulletin 76, 105-110. DOI: 10.1037/h0031322.

Zielonka, P., 2004. Technical analysis as the representation of typical cognitive biases. International Review of Financial Analysis 13, 217-225. DOI: 10.1016/j.irfa.2004.02.007. 